\title{
Transcriptome-derived stromal and immune scores infer clinical outcomes of patients with cancer
}

\author{
WEI LIU ${ }^{1,2}$, HUA YE $^{3}$, YING-FU LIU ${ }^{4}$, CHAO-QUN XU ${ }^{1}$, YUE-XIAN ZHONG ${ }^{1}$, TIAN TIAN ${ }^{1}$, \\ SHI-WEI MA ${ }^{1}$, HUAN TAO ${ }^{1}$, LING LI $^{1}$, LI-CHUN XUE ${ }^{1}$ and HUA-QIN HE ${ }^{1}$ \\ ${ }^{1}$ School of Life Sciences, Fujian Agriculture and Forestry University, Fuzhou, Fujian 350002; ${ }^{2}$ Department of Pathology, \\ Human Centrifuge Medical Training Center, Institute of Aviation Medicine of Chinese PLA Air Force, Beijing 100089; \\ ${ }^{3}$ Department of Gastroenterology, Ningbo Medical Treatment Center Lihuili Hospital, Ningbo, Zhejiang 315040; \\ ${ }^{4}$ Department of Cell Biology, Logistics University of Chinese Armed Police Forces, Tianjin 300309, P.R. China
}

Received December 8, 2016; Accepted November 29, 2017

DOI: $10.3892 / \mathrm{ol} .2018 .7855$

\begin{abstract}
The stromal and immune cells that form the tumor microenvironment serve a key role in the aggressiveness of tumors. Current tumor-centric interpretations of cancer transcriptome data ignore the roles of stromal and immune cells. The aim of the present study was to investigate the clinical utility of stromal and immune cells in tissue-based transcriptome data. The 'Estimation of STromal and Immune cells in MAlignant Tumor tissues using Expression data' (ESTIMATE) algorithm was used to probe diverse cancer datasets and the fraction of stromal and immune cells in tumor tissues was scored. The association between the ESTIMATE scores and patient survival data was asessed; it was indicated that the two scores have implications for patient survival, metastasis and recurrence. Analysis of a colorectal cancer progression dataset revealed that decreased levels immune cells could serve an important role in cancer progression. The results of the present study indicated that trasncriptome-derived stromal and immune scores may be a useful indicator of cancer prognosis.
\end{abstract}

\section{Introduction}

Cancer is a genetic disease characterized by genomic abnormalities that alter the transcriptome and influence the pathways that control proliferation and survival (1). The application of next-generation sequencing technology and single-cell sequencing in oncology has provided evidence that cellular heterogeneity is common in cancer (2). In the majority of tumor studies, key information may be disregarded owing to the tissue-centric nature of research. Malignant

Correspondence to: Professor Hua-Qin He or Dr Li-Chun Xue, School of Life Sciences, Fujian Agriculture and Forestry University, 15 Shangxiadian Road, Fuzhou, Fujian 350002, P.R. China

E-mail: hehq3@fafu.edu.cn

E-mail: 22125492@qq.com

Key words: immune cells, recurrence, stromal cells, survival solid tumor tissues consist mainly of tumor cells, but also contain tumor-associated stromal, immune and vascular cells. Although non-tumor cells constitute a relatively small proportion of the cancer tissue, their role as potent tumor promoters has been previously indicated (3). A previous study, using a network approach to identify the functional gene modules in cancer cells, verified the presence of immune, stromal and vascular gene modules in cancer tissues (4).

The majority of genomic and transcriptomic studies into cancer do not explicitly consider genetic heterogeneity, and the generated inferences usually refer to mixed cell populations (5). However, the experimental isolation of single cells from tissues is expensive and may affect cell physiology. Additionally, the single-cell sequencing of a large cohort is unrealistic. An efficient solution to this limitation may be the de-convolution of genomic data from heterogeneous samples. Publicly available transcriptome databases can provide resources that allow for this type of analysis. To date, only one method, referred to as 'Estimation of STromal and Immune cells in MAlignant Tumors using Expression data' (ESTIMATE) has been described that can be used to score the stromal and immune fraction in transcriptomic data of cancer tissue $(5,6)$. However, to the best of our knowledge, the association of the proportion of immune and stromal fraction with patient survival has not been thoroughly investigated.

Several studies have examined the microenvironment-associated transcriptional tumor profile using transcriptomic data. Calon et al (7) identified transforming growth factor- $\beta$ (TGF- $\beta$ ) response signatures in tumor-associated stromal cells that could predict disease relapse in colorectal cancer (CRC). Cheng et al (8) used principle component analysis and clustering methods to identify a signature of stromal activation that was associated with late recurrence in breast cancer. Teschendorff et al (9) described an immune response gene expression module associated with a good prognostic subtype in estrogen receptor negative breast cancer. Finak et al (10) used laser capture microdissection (LCM) to compare the gene expression profiles of tumor stroma from primary breast tumors and derived signatures that were strongly associated with the clinical outcome by clustering. Isella et al (11) used Gene Set Enrichment Analysis (GSEA) and examined the gene 
signatures of subtypes for expression in stromal cell subpopulations vs. CRC cells. Wu et al (12) identified a stromal gene super-module associated with gastric cancer patient survival using gene co-expression network analysis. Furthermore, extensive experimental research has indicated the role served by stromal and immune cells in breast cancer $(8,10,13)$, CRC $(7,11,14)$, lymphoma (15) and drug resistance $(16,17)$.

Transcriptome-based subtyping of cancer identifies different subtypes by clustering; however, non-tumor components are usually ignored (18). The ESTIMATE algorithm scores stromal and immune cells that form the major non-tumor components of tumor samples. In the present study, the scoring of stromal and immune cells in healthy and cancerous tissues, as well as in disease prognosis and drug resistance was investigated. The scores were associated with the clinicopathological characteristics of various cancer types and chemotherapeutic drug resistance. The results of the present study indicated that ESTIMATE could be used as a metric for patient prognosis assessment.

\section{Materials and methods}

Microarray datasets of healthy and disease tissue. The normal tissue dataset GSE45878 and cancer tissue dataset GSE2109 were obtained from the Gene Expression Omnibus (GEO) database (www.ncbi.nlm.nih.gov/geo/). A validation RNA-Seq dataset E-MTAB-2836 from 32 different normal tissues was downloaded from EBI ArrayExpress database (www.ebi.ac.uk/arrayexpress/experiments/ E-MTAB-2836/) (19).

ESTIMATE algorithm. Stromal and immune scores were calculated by the ESTIMATE package in R (version 2.15.3) (20). ESTIMATE algorithm exploits the unique properties of the transcriptional profiles of cancer samples to infer tumor cellularity and identify the infiltrating normal cells (6). Five rounds of gene filtering identified two distinct gene signatures: i) A 'stromal signature' that indicates the stroma, and ii) an 'immune signature' that represents the infiltration of immune cells in tumor tissue. ESTIMATE outputs stromal, immune and ESTIMATE scores by performing single-sample GSEA. For a given sample, gene expression values were rank-normalized and rank-ordered. The empirical cumulative distribution functions of the signature genes and the remaining genes were calculated. A value of statistical significance was calculated by integrating the difference between the empirical cumulative distribution function, which is similar to the one used in GSEA, but based on absolute expression rather than differential expression (6).

Survival analysis. The breast cancer (GSE31448), CRC (GSE17538, GSE41258, GSE39396), Ewing's sarcoma (GSE17679), glioma (GSE16011), hepatocellular carcinoma (GSE20140), leukemia (GSE12417), lung cancer (GSE3141), lymphoma (GSE10846), melanoma (GSE65904) and ovarian cancer (GSE32062) datasets, and the respective clinical information were obtained from the GEO repository.

For metastasis and relapse analysis, the sarcoma (GSE21050), breast cancer (GSE1456), hepatocellular carcinoma (GSE10140), gastric cancer (GSE26253) and prostate cancer (GSE46691) datasets were obtained from the GEO database. The Cancer Genome Atlas (TCGA) expression dataset was obtained from Firebrowse at Broad Institute of the Massachusetts Institute of Technology \& Harvard (firebrowse.org/).

Statistical analysis. The ESTIMATE scores for each dataset were calculated and patients were divided into two equal groups of high or low ESTIMATE score by median split. The ESTIMATE scores were normalized prior to Cox proportional hazards multivariate analysis. Overall survival time curves were plotted using the Kaplan-Meier method. Distributions of overall survival were compared using the log-rank test. Metastasis-free survival curves were plotted similarly for the samples that metastasis information was available.Furthermore, TCGA datasets' scores were associated with clinical information using Cox proportional hazards multivariate analysis. All analyses were conducted using SPSS software (version 17.0; SPSS, Inc., Chicago, IL, USA). The concordance index was used to indicate the probability that a patient with decreased survival time is associated with a high value of a predictor. It was estimated using the rms R package (21). ESTIMATE score differences between two groups were assessed using unpaired two-tailed t-tests in Microsoft Excel. $\mathrm{P}<0.05$ was considered to indicate a statistically significant difference. For multiple comparisons, Bonferroni corrections were applied following analysis of variance, and $\mathrm{P}<0.05 /$ number of tests were used as significance threshold. The correlation between lymphoblastoid cell line immune score and 5-FU treatment response was calculation by Pearson's correlation analysis in SPSS.

\section{Results}

Stromal and immune scores in healthy and disease tissues. To investigate the difference in ESTIMATE scores between healthy and malignant tissues, two public microarray datasets, GSE45878 and GSE2109, were analyzed (Fig. 1). Among normal tissues, adipose had the highest stromal score, whereas brain had the lowest; lung had the highest immune score whereas brain had the lowest. The present findings are consistent with the results from an RNA-sequencing dataset (E-MTAB-2836). Regarding malignant tissues, almost all of them presented a positive average ESTIMATE score: Pancreas had the highest stromal score and prostate the lowest, whilst testis had the highest immune score and prostate the lowest. Notably, normal pancreas had a low stromal score and normal testis had a low immune score (data not shown), whereas cancerous pancreas had a high stromal score and cancerous testis had a high immune score. Leukemia presented an extremely narrow range of scores, indicating the robust performance of the ESTIMATE algorithm.

Stromal and immune scores as a potential prognostic marker for multiple types of cancer. We subsequently hypothesized that as the two scores represent common malignancy features, they may be used as potential markers for cancer prognosis. The prognostic efficiency of stromal and immune scores in breast cancer, CRC, Ewing's sarcoma, glioma, hepatocellular carcinoma, leukemia, lymphoma, 

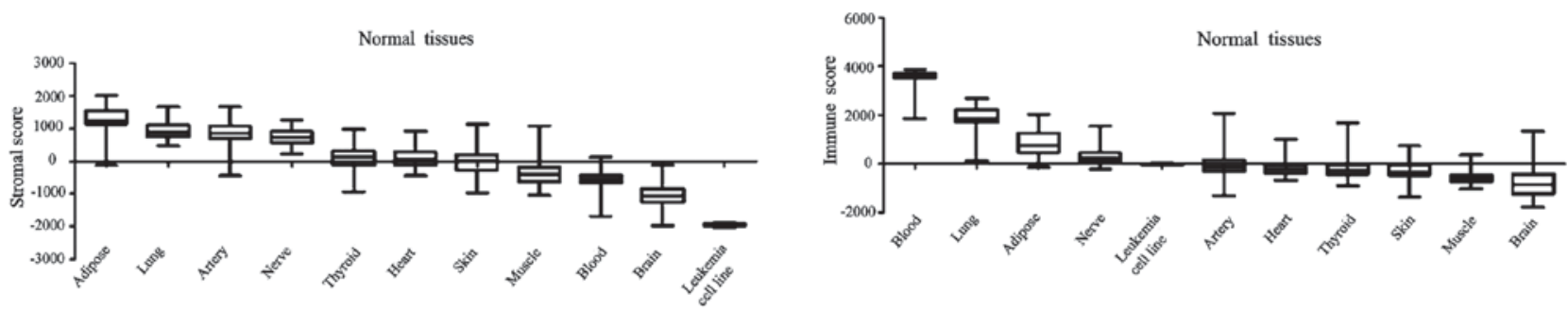

Cancerous tissues
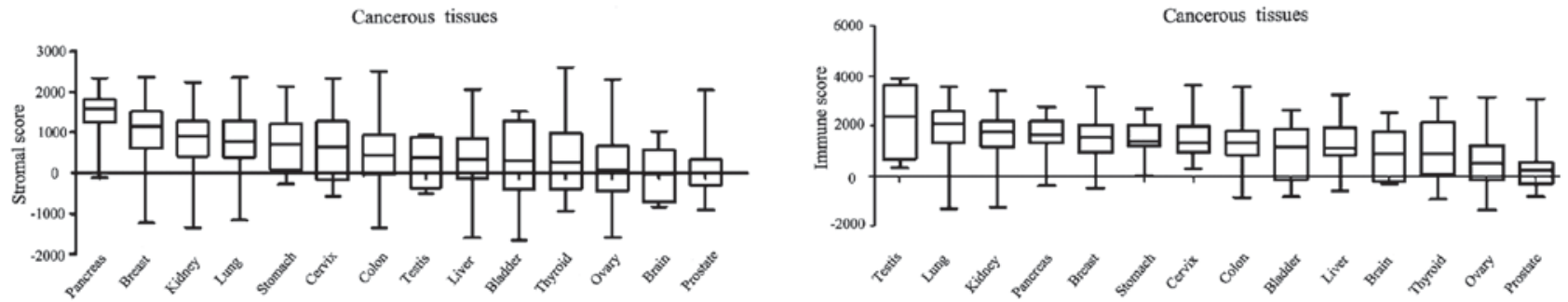

Figure 1. Stromal and immune scores in healthy and malignant tissues.
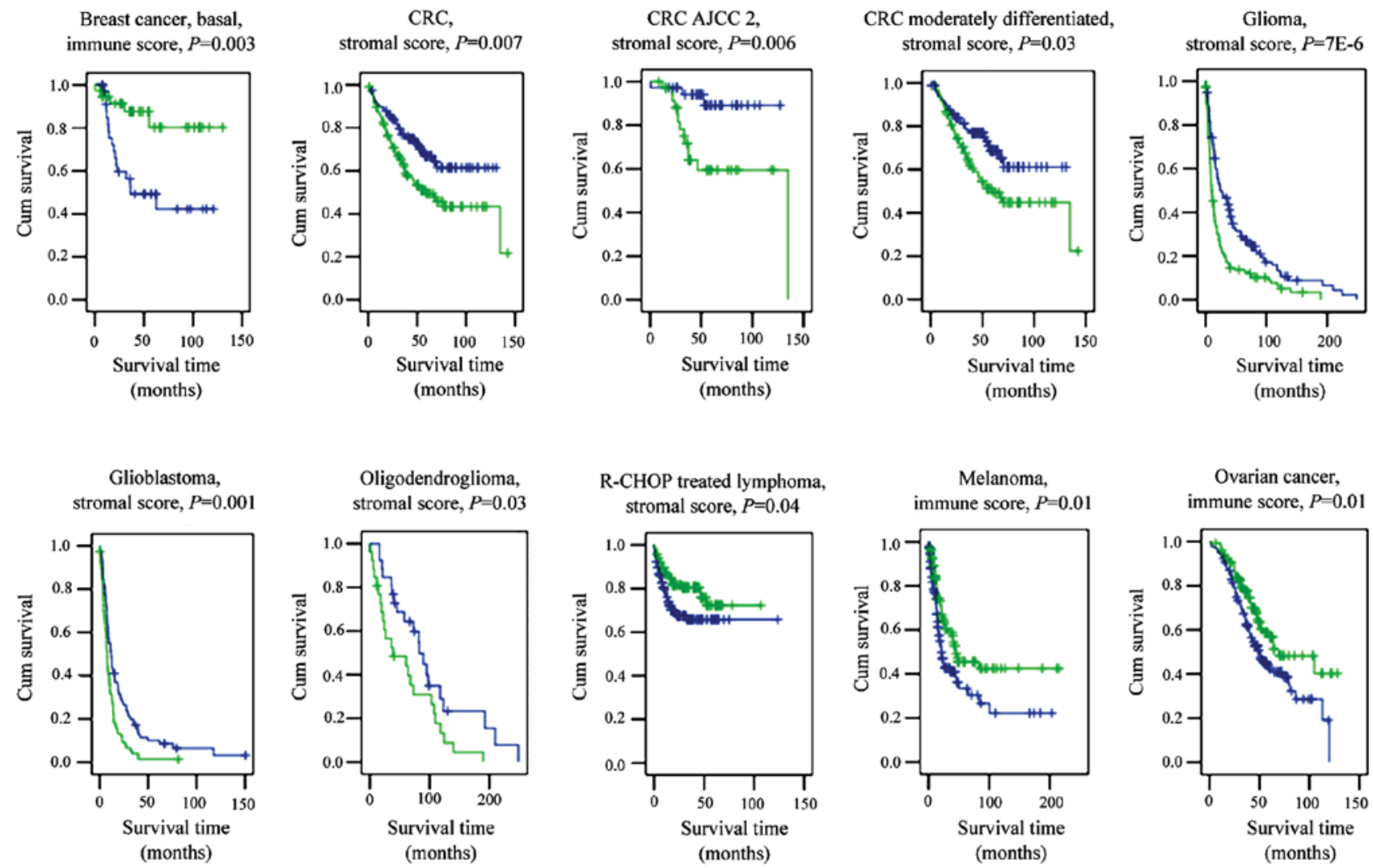

Figure 2. Stromal and immune scores are predictive of patient survival in several types of cancer. Kaplan-Meier curves of patients with breast cancer, CRC, glioma, lymphoma, melanoma and ovarian cancer. P-values were generated using the log-rank test. Blue lines indicate low scores; green lines indicate high scores. AJCC, American Joint Committee on Cancer; CRC, colorectal cancer; R-CHOP, rituximab, cyclophosphamide, hydroxydaunorubicin, oncovin and prednisone; Cum, cumulative.

lung cancer, melanoma, ovarian cancer, prostate cancer and sarcoma was investigated. Survival curves were plotted following patient categorization in groups of high and low ESTIMATE scores. Notably, the two scores were indicative of patient survival in multiple types of cancer (Fig. 2). Indicatively, the immune score separated patients with long and short survival in ovarian cancer and melanoma. Additionally, the stromal score signified the survival time of patients with CRC and glioma. Furthemore, the scores indicated survival rates within specific subtypes of cancer. For instance, the stromal score indicated the survival rate of patients with stage 2 American Joint Committee on Cancer (AJCC) CRC (22), moderately differentiated CRC, oligodendroglioma, glioblastoma and served a prognostic role in patients with rituximab, cyclophosphamide, doxorubicin, vincristine and prednisone-treated lymphoma. The immune 
Table I. Multivariate Cox regression of overall survival and metastasis data in several types of cancer.

\begin{tabular}{lllcc}
\hline Data source & \multicolumn{1}{c}{ Disease } & \multicolumn{1}{c}{ Variable } & P-value & Estimated hazard ratio \\
\hline Gene Expression Omnibus & Colorectal cancer survival & Stromal score & 0.008 & 1.487 \\
& Breast cancer survival & Immune score & 0.001 & 0.544 \\
& Gastric cancer survival & Stromal score & 0.002 & 1.314 \\
& Glioma survival & Stromal score & 0.009 & 1.437 \\
& Melanoma survival & Immune score & 0.000 & 0.541 \\
& Ovarian cancer survival & Stromal & 0.033 & 1.327 \\
& & Immune score & 0.001 & 0.649 \\
The Cancer Genome Atlas & Sarcoma metastasis & Stromal score & 0.002 & 0.702 \\
& Breast cancer survival & Stromal score & 0.040 & 1.278 \\
& & Immune score & 0.046 & 0.744 \\
& Cervical squamous cell carcinoma survival & Stromal score & 0.026 & 1.456 \\
& & Immune score & 0.008 & 0.611 \\
& Skin cutaneous melanoma survival & Immune score & 0.002 & 0.685 \\
\hline
\end{tabular}

score indicated the survival time of patients with basal breast cancer.

The stromal score separated patients with early relapse from those with late relapse in basal breast cancer, and differentiated between patients with early metastasis and late metastasis in sarcoma (Fig. 3). Notably, the immune score of hepatocellular carcinoma-adjacent hepatitis/cirrhotic liver tissue was found to be indicative of disease recurrence.

Certain GEO and TCGA datasets that contained additional clinical information were further analyzed by multivariate analysis. The immune score indicated the survival rate of patients with breast cancer, melanoma and ovarian cancer following adjustment for clinical parameters (Table I). Additionally, the stromal score indicated the survival rate of patients with CRC and glioma, and predicted cancer recurrence and metastasis in patients with sarcoma following adjustment for clinical parameters (Table I). Furthermore, the clinical implication of the immune score in breast cancer and melanoma was also validated in the TCGA dataset.

Stromal and immune scores predict CRC progression. The significance of the cancer microenvironment in tumor progression has been repeatedly indicated. Stromal and immune cells are major non-tumor components of cancer. Even though the stromal score is predictive of survival in patients with AJCC stage $2 \mathrm{CRC}$, the immune score indicated a progression from polyp to CRC. The average immune scores in normal colon mucosa, polyp, primary CRC and metastatic CRC were calculated as 1,203, 488, 887 and 500, respectively. However, no statistically significant difference between the normal colon mucosa and polyp tissue was observed. The immune score was significantly lower in primary and metastatic CRC than in normal colon or polyp tissue. An association between the downregulation of immune system-associated genes and metastasis in CRC has been previously reported (23). The immune score is significantly lower in p53 mutant patients $(\mathrm{P}=0.02)$ in the GSE41258 dataset. It has been demonstrated that p53 regulates immunological activities (24), indicating that $\mathrm{p} 53$ has a possible regulatory role in CRC progression.

Gene expression analysis of specific cellular populations isolated from CRC patients revealed that fibroblasts and leukocytes have the highest stromal and immune scores among endothelial cells, epithelial cells, fibroblasts and leukocytes (Fig. 4). These results indicate the robustness of the ESTIMATE algorithm.

Implication of drug resistance by immune score. The anticancer activity of fluorouracil (5-FU) involves the restoration of T-cell immunity (25). The GSE11582 dataset includes the response of lymphoblastoid cell lines treated with a range of 5-FU concentrations. Pearson's correlation analysis revealed that the immune score was positively correlated with 5-FU treatment response $(\mathrm{R}=0.2, \mathrm{P}=0.008)$.

\section{Discussion}

To the best of our knowledge, this is the first study in which ESTIMATE scoring was used to differentiate between tissues. Stromal and immune scores were associated with the clinical outcome of the patient and chemotherapy drug resistance. The prognostic value of the two scores was validated using multiple microarray platforms. Either the stromal or the immune score was associated with patient survival, relapse and metastasis in multiple types of cancer. Furthermore, the two scores were associated with chemotherapeutic drug response. The present study has indicated a microenvironment view of tissue-based tumor transcriptomic data and highlighted the contribution of stromal and immune cells in carcinogenesis. However, the precise molecular mechanism underlying this phenomenon should be investigated in future studies.

Tissues are composed of a mixture of cell types. Currently, tissue-based transcriptomic data do not reflect the information from multiple cell types. Even though a number of technological approaches, including flow cytometry and LCM, have been developed, their application in cancer research is limited owing to the expensive cost. Therefore, it is crucial to study 
Breast cancer, stromal score, $P=0.03$

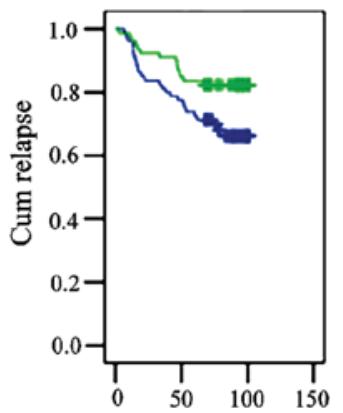

Relapse free survival time (months)
$\mathrm{GC}$, stromal score, $P=0.004$

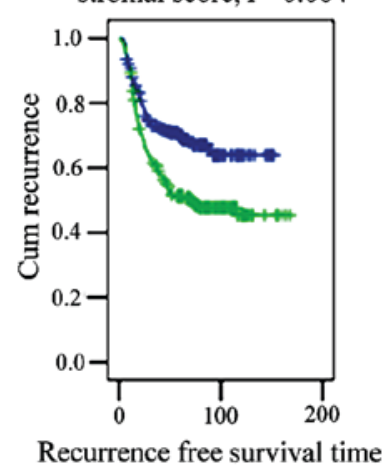

(months)
HCC adjacent, immune score, $P=0.04$

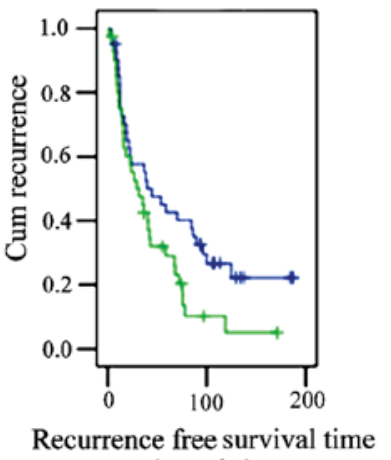

(months)
Sarcoma, stromal score, $P=0.001$

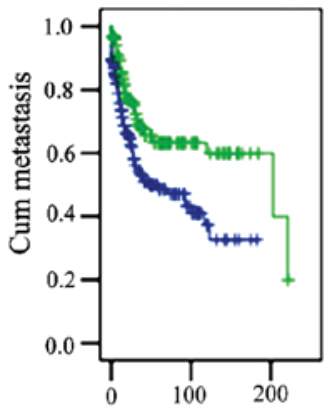

Metastasis free survival time

(months)

Figure 3. Stromal and immune scores are indicative of patient recurrence and metastasis. Kaplan-Meier curves of patients with breast cancer, gastric cancer, hepatocellular carcinoma and sarcoma. P-values were generated using the log-rank test. Blue lines indicate low scores; green lines indicate high scores. GC, gastric cancer; HCC, hepatocellular carcinoma; Cum, cumulative.
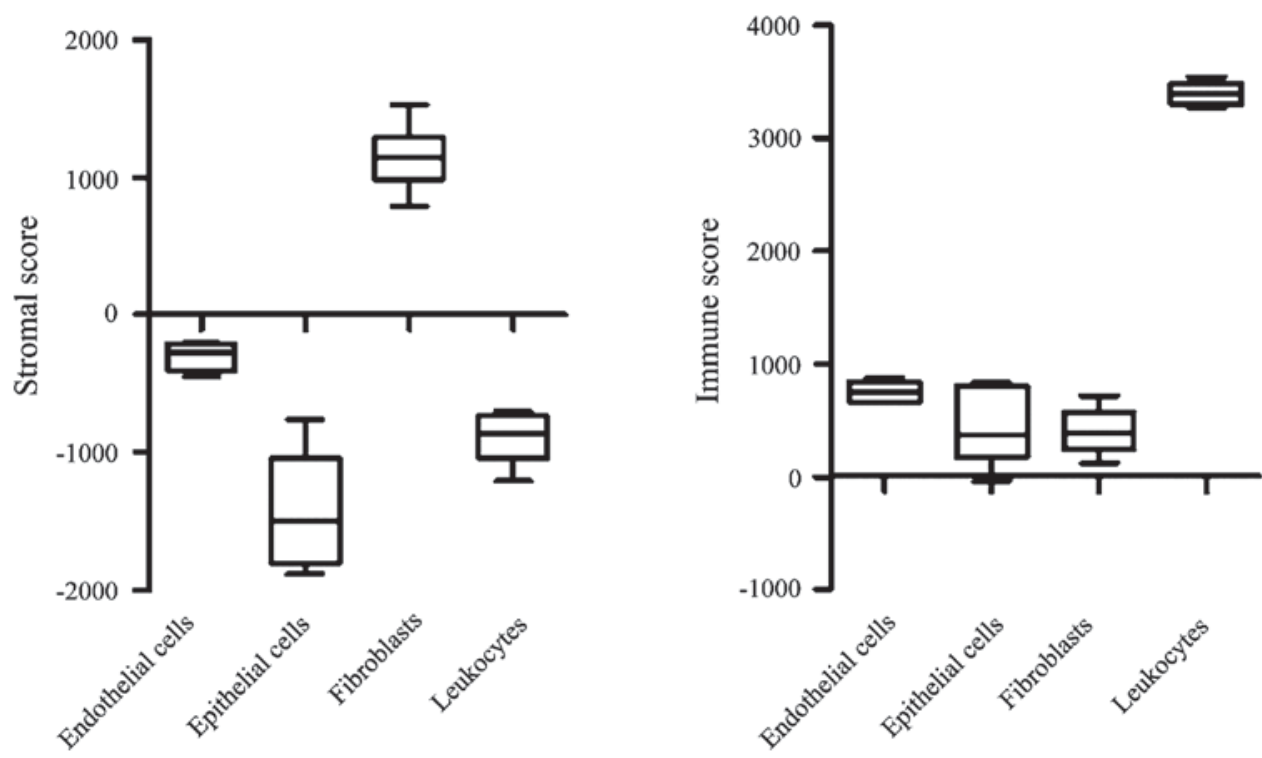

Figure 4. Stromal and immune scores in distinct cellular populations derived from patients with colorectal carcinoma.

the information hidden in available public datasets. Functional modules in cancer transcriptomic datasets have been previously identified by gene co-expression network analysis (4). In the present study, two major components of the cancer microenvironment, stromal and immune cells, were investigated, and it was demonstrated that the ESTIMATE scores for these components could predict patient clinical outcomes. For instance, the stroma score was identified as a predictor for survival in patients with gastric cancer (a lower stroma score was indicative of better patient prognosis). Notably, the ESTIMATE-derived stromal score was found to be more efficient than the recently described stromal super-module-based method (12). However, it should be mentioned that the stroma might serve distinct roles in different tissues. In the present study it was demonstrated that a higher stroma score indicated later relapse in breast cancer, which is consistent with a clustering-derived gene signature method $(8,10)$. The immune score was also found to predict survival in basal breast cancer, in agreement with a previous study that used the
Profile Analysis using the Clustering and Kurtosis method (9). Similarly, a higher immune score was associated with a longer survival time in melanoma and ovarian cancer, but earlier recurrence in hepatocellular carcinoma-adjacent tissue. The degree of tumour-infiltrating lymphocytes in particular activated CD8+ T-cells, within melanoma positively correlates with better prognosis (26). The decreased recruitment of tumor-infiltrating lymphocytes may lead to poor prognosis in high-risk ovarian cancer patients (27). It has been previously demonstrated that the poor prognostic signature in hepatocellular carcinoma-adjacent tissue involved genes associated with inflammation, including interferon signaling and activation of nuclear factor- $\mathrm{\kappa B}$ and tumor necrosis factor (28). These results indicated that non-tumor liver tissue could serve a prognostic role in patients with early-stage disease. These results may suggest the dual host-protective and tumor-promoting roles of immune cells in different tumor types $(29,30)$.

The results of the present study indicated that research into microenvironment-associated cells is warranted in 
patients with cancer. Understanding the effect of the microenvironment on drug sensitivity may improve the efficiency of targeted therapies (31). For instance, an association has been demonstrated between the initiation of metastasis in CRC and the TGF- $\beta$ stromal program (7). Even though the ESTIMATE algorithm is based on cancer tissue data, it was found to be effective in assessing cellular data as well (Fig. 4). In the present study, the ESTIMATE algorithm was used on cell line data, identifying a positive correlation between 5-FU treatment response and the immune score and indicating the potential mechanism of the drug (25). A lower immune score was observed in p53-mutant CRC patients, indicating that 5-FU may not be the optimal treatment choice for $\mathrm{p} 53$ mutant patients. Indeed, several clinical studies have reported that CRC patients with wild-type p53 benefit from 5-FU-based chemotherapy, but those with mutant TP53 do not $(32,33)$. Thus, robust patient stratification using microenvironment data may aid the development and application of cancer therapies (34).

It is reasonable to apply the ESTIMATE scoring to a specific tissue-based transcriptomic dataset, as the sampling criteria are identical for every specimen within a study. To the best of our knowledge, the present study is the first to demonstrate that ESTIMATE scores are indicative of patient survival, relapse, metastasis and chemotherapeutic drug resistance. The two scores may have a prognostic value, indicating that stromal and immune cells contribute to tumor clinical outcome. It was further demonstrated that immune cells were associated with CRC development and that the ESTIMATE scores may become useful indicators of tissue-based patient prognosis.

\section{Acknowledgements}

The present study was supported by the National Natural Science Foundation of China (grant nos. 81400617 and 81502091), the National Natural Science Foundation of Zhejiang Provincial China (grant no. LQ14H030001) and the Ningbo Natural Science Foundation (grant no. 2013A610232).

\section{References}

1. Klausner RD: The fabric of cancer cell biology-Weaving together the strands. Cancer Cell 1: 3-10, 2002.

2. Navin N, Kendall J, Troge J, Andrews P, Rodgers L, McIndoo J, Cook K, Stepansky A, Levy D, Esposito D, et al: Tumour evolution inferred by single-cell sequencing. Nature 472: 90-94, 2011.

3. Bissell MJ and Hines WC: Why don't we get more cancer? A proposed role of the microenvironment in restraining cancer progression. Nat Med 17: 320-329, 2011.

4. Liu W, Li L and Li W: Gene co-expression analysis identifies common modules related to prognosis and drug resistance in cancer cell lines. Int J Cancer 135: 2795-2803, 2014

5. Yadav VK and De S: An assessment of computational methods for estimating purity and clonality using genomic data derived from heterogeneous tumor tissue samples. Brief Bioinform 16: 232-241, 2015

6. Yoshihara K, Shahmoradgoli M, Martinez E, Vegesna R, Kim H, Torres-Garcia W, Treviño V, Shen H, Laird PW, Levine DA, et al: Inferring tumour purity and stromal and immune cell admixture from expression data. Nat Commun 4: 2612, 2013.

7. Calon A, Espinet E, Palomo-Ponce S, Tauriello DV, Iglesias M, Céspedes MV, Sevillano M, Nadal C, Jung P, Zhang XH, et al: Dependency of colorectal cancer on a TGF- $\beta$-driven program in stromal cells for metastasis initiation. Cancer Cell 22: 571-584, 2012.
8. Cheng Q, Chang JT, Gwin WR, Zhu J, Ambs S, Geradts J and Lyerly HK: A signature of epithelial-mesenchymal plasticity and stromal activation in primary tumor modulates late recurrence in breast cancer independent of disease subtype. Breast Cancer Res 16: 407, 2014.

9. Teschendorff AE, Miremadi A, Pinder SE, Ellis IO and Caldas C: An immune response gene expression module identifies a good prognosis subtype in estrogen receptor negative breast cancer. Genome Biol 8: R157, 2007.

10. Finak G, Bertos N, Pepin F, Sadekova S, Souleimanova M, Zhao H, Chen H, Omeroglu G, Meterissian S, Omeroglu A, et al: Stromal gene expression predicts clinical outcome in breast cancer. Nat Med 14: 518-527, 2008.

11. Isella C, Terrasi A, Bellomo SE, Petti C, Galatola G, Muratore A, Mellano A, Senetta R, Cassenti A, Sonetto C, et al: Stromal contribution to the colorectal cancer transcriptome. Nat Genet 47: 312-319, 2015.

12. Wu Y, Grabsch H, Ivanova T, Tan IB, Murray J, Ooi CH, Wright AI, West NP, Hutchins GG, Wu J, et al: Comprehensive genomic meta-analysis identifies intra-tumoural stroma as a predictor of survival in patients with gastric cancer. Gut 62: 1100-1111, 2013.

13. Bianchini G, Qi Y, Alvarez RH, Iwamoto T, Coutant C, Ibrahim NK, Valero V, Cristofanilli M, Green MC, Radvanyi L, et al: Molecular anatomy of breast cancer stroma and its prognostic value in estrogen receptor-positive and -negative cancers. J Clin Oncol 28: 4316-4323, 2010.

14. Calon A, Lonardo E, Berenguer-Llergo A, Espinet E, Hernando-Momblona X, Iglesias M, Sevillano M, Palomo-Ponce S, Tauriello DV, Byrom D, et al: Stromal gene expression defines poor-prognosis subtypes in colorectal cancer. Nat Genet 47: 320-329, 2015.

15. Meyer PN, Fu K, Greiner T, Smith L, Delabie J, Gascoyne R, Ott G, Rosenwald A, Braziel R, Campo E, et al: The stromal cell marker SPARC predicts for survival in patients with diffuse large B-cell lymphoma treated with rituximab. Am J Clin Pathol 135: 54-61, 2011.

16. Goossens N, Hoshida Y and Aguirre-Ghiso JA: Origin and interpretation of cancer transcriptome profiling: The essential role of the stroma in determining prognosis and drug resistance. EMBO Mol Med 7: 1385-1387, 2015.

17. Quigley DA and Kristensen V: Predicting prognosis and therapeutic response from interactions between lymphocytes and tumor cells. Mol Oncol 9: 2054-2062, 2015.

18. Rhodes DR and Chinnaiyan AM: Integrative analysis of the cancer transcriptome. Nat Genet 37 (Suppl): S31-S37, 2005.

19. Uhlén M, Fagerberg L, Hallström BM, Lindskog C, Oksvold P, Mardinoglu A, Sivertsson Å, Kampf C, Sjöstedt E, Asplund A, et al: Proteomics. Tissue-based map of the human proteome. Science 347: 1260419, 2015.

20. Team RC: R A language and environment for statistical computing. Vienna Austria R Foundation for Statistical Computing. Available from http //www.R-project.org. Accessed February 10, 2015.

21. Harrell FE Jr: rms: Regression Modeling Strategies. R package version 4.1-3. Available from http://CRAN.R-project.org/package=rms, 2014.

22. Edge SB and Compton CC: The American Joint Committee on Cancer: The 7th edition of the AJCC cancer staging manual and the future of TNM. Ann Surg Oncol 17: 1471-1474, 2010.

23. Fehlker M, Huska MR, Jöns T, Andrade-Navarro MA and Kemmner W: Concerted down-regulation of immune-system related genes predicts metastasis in colorectal carcinoma. BMC Cancer 14: 64, 2014.

24. Menendez D, Shatz M and Resnick MA: Interactions between the tumor suppressor p53 and immune responses. Curr Opin Oncol 25: 85-92, 2013.

25. Bracci L, Schiavoni G, Sistigu A and Belardelli F: Immune-based mechanisms of cytotoxic chemotherapy: Implications for the design of novel and rationale-based combined treatments against cancer. Cell Death Differ 21: 15-25, 2014.

26. Tan LY, Martini C, Fridlender ZG, Bonder CS, Brown MP and Ebert LM: Control of immune cell entry through the tumour vasculature: A missing link in optimising melanoma immunotherapy? Clin Trans Immunol 6: e134, 2017.

27. Yoshihara K, Tsunoda T, Shigemizu D, Fujiwara H, Hatae M, Fujiwara H, Masuzaki H, Katabuchi H, Kawakami Y, Okamoto A, et al: High-risk ovarian cancer based on 126-gene expression signature is uniquely characterized by downregulation of antigen presentation pathway. Clin Cancer Res 18: 1374-1385, 2012. 
28. Hoshida Y, Villanueva A, Kobayashi M, Peix J, Chiang DY, Camargo A, Gupta S, Moore J, Wrobel MJ, Lerner J, et al: Gene expression in fixed tissues and outcome in hepatocellular carcinoma. N Engl J Med 359: 1995-2004, 2008.

29. Schreiber RD, Old LJ and Smyth MJ: Cancer immunoediting: Integrating immunity's roles in cancer suppression and promotion. Science 331: 1565-1570, 2011.

30. Linsley PS, Chaussabel D and Speake C: The relationship of immune cell signatures to patient survival varies within and between tumor types. PLoS One 10: e0138726, 2015.

31. Donner A: Drug resistance: The stroma's contribution. Nat Chem Biol 8: 739, 2012.

32. Russo A, Bazan V, Iacopetta B, Kerr D, Soussi T and Gebbia N TP53-CRC Collaborative Study Group: The TP53 colorectal cancer international collaborative study on the prognostic and predictive significance of p53 mutation: Influence of tumor site, type of mutation, and adjuvant treatment. J Clin Oncol 23 : $7518-7528,2005$
33. Boyer J, McLean EG, Aroori S, Wilson P, McCulla A, Carey PD, Longley DB and Johnston PG: Characterization of p53 wild-type and null isogenic colorectal cancer cell lines resistant to 5-fluorouracil, oxaliplatin, and irinotecan. Clin Cancer Res 10: 2158-2167, 2004.

34. Cohen RL and Settleman J: From cancer genomics to precision oncology-tissue's still an issue. Cell 157: 1509-1514, 2014.

(c) (i) () $($ This work is licensed under a Creative Commons

EY NG ND Attribution-NonCommercial-NoDerivatives 4.0 International (CC BY-NC-ND 4.0) License. 